\title{
Author response: use of bioartificial dermal regeneration template for skin restoration in combat casualty injuries
}

Response to: Leclère FM \& Casoli V. Use of bioartificial dermal regeneration template for skin restoration in combat casualty injuries. Regen. Med. 11(4), 359-360 (2016).

First draft submitted: 11 April 2016; Accepted for publication: 21 April 2016; Published online: 26 May 2016

The authors appreciate the positive review and response regarding our recent published work on dermal regenerate templates (DRTs) in combat casualty and reconstructive care. We agree with the various advantages for the use of DRTs summarized as follows: utilization for soft tissue replacement and improved contour restoration after tissue loss; readily available off-the shelf tissue substitute which can lessen donor site morbidity of certain flaps/tissue replacement options although primary- or secondary-staged skin grafting will be required; reduction in complexity with regard to technical aspects of DRT application compared with more complex flap design and rapid restoration of soft tissue defect with relative early return to activities of daily living.

Our group also agrees with the additional advantages described as follows. The application of a DRT can be used for coverage over an exposed microvascular or rotational flap pedicle for short-term or long-term coverage. This technique has been employed in a few cases of both upper as well as lower extremity limb salvage and residual limb length preservation surgeries as a temporary or permanent measure when a tight soft tissue envelope has been encountered over a flap vascular pedicle to reduce the external forces or pressure on the vascular arterial or venous system. Depending on the situation, we have either proceeded with secondary skin grafting over the DRT once it has incorporated or simply 'bury' the incorporated DRT after removing the silicone layer by way of an adjacent tissue transfer, flap advancement or delayed primary closure over the prior flap pedicle. This scenario is often encountered during cases where significant soft tissue edema occurs and sufficient soft tissue redundancy is not present to permit a tension-free closure or coverage over the vascular pedicle. Thus, we have placed a DRT over the exposed vascular structures to lessen the tension over these structures while also allowing for ease in detection of a hematoma or fluid collection around the pedicle. However, hematomas or fluid collections that caused vascular/pedicle compromise were not common in our experiences. This situation may have been avoided by our group via 'pie-crusting' or placing perforations within the DRT in areas not directly over the vascular pedicle, avoidance of a negative pressure system or bolster dressing over the DRT in these specific cases to avoid direct compression over the pedicle site, and application of a nonstick silver dressing such as Acticoat ${ }^{\mathrm{TM}}$ Flex 3 or Flex 7 or a Restore ${ }^{\circledR}$ Contact Layer with Silver and TRIACT TM Technology which were the two most common silver dressings applied in these cases.

Other advantages to the use of DRTs that our group has experienced include: decreased erosion and skin graft shearing in residual limbs at the prosthetic and soft tissue interface when DRTs are used in combination with sheet skin grafts compared with those
Ian Valerio*,1 \& Scott Tintle ${ }^{2}$

'The Ohio State University Wexner Medical Center, Columbus, OH, USA ${ }^{2}$ Walter Reed National Military Medical Center, Bethesda, MD, USA *Author for correspondence: iv_cwru@yahoo.com 
residual limbs where skin grafts were used in isolation; less contracture of skin grafts used over DRTS as compared with skin grafts used in isolation when applied to extremity and/or joint surface wounds and many patients who receive a DRT and negative pressure dressing can be managed easily within an outpatient setting. After surgical debridement of the extremity wound, DRT placement and securing to the wound bed with sutures and application of a negative pressure dressing system, the patient can be discharged from the inpatient setting with weekly follow-up for dressing changes until DRT incorporation and secondary skin grafting procedure. This benefit can aid in reducing costs associated with a lengthy inpatient hospital stay.

Another benefit of use of DRTs has been in those cases where secondary surgical procedures are indicated or necessary. Specifically, in our experience, DRT and skin graft constructs tolerate tenolysis, tendon work, and certain other reconstructive procedures with less wound healing and breakdown issues when compared to those cases where skin grafts alone were used in prior reconstructions. Heterotopic ossification (HO) is an abnormal condition of bone formation within soft tissues, joint, or within residual limbs which has been shown to cause functional issues in a high rate of combat injured patients. In such cases where $\mathrm{HO}$ excision was indicated or necessary, those reconstructions that previously utilized a DRT and skin graft construct more often tolerated secondary $\mathrm{HO}$ excisional procedures better when compared to those cases where skin grafting alone was employed in their prior reconstructions. Those HO cases where DRT and skin graft constructs were performed at their prior definitive coverage procedure were much more commonly able to be closed with re-advancement or primary closure of the construct over the $\mathrm{HO}$ extirpative defect as compared to those cases where skin grafts alone were utilized. The DRT and skin graft combination showed the ability to provide sufficient soft tissue for closure as compared to that of the skin graft group which often had significantly higher erosion and fragmentation of their skin grafts from the underlying $\mathrm{HO}$ overgrowth and during the $\mathrm{HO}$ extirpation surgery. Finally, DRT and skin graft constructs also tolerate fat grafting procedures for contour restoration or soft tissue augmentation of the underlying soft tissue as compared to skin grafting alone.

The main disadvantage to use of DRTs is that these tissue substitutes can be rather expensive. However, when applied in the correct settings or situations, DRTs can be quite cost effective, may avoid certain preventable or unnecessary morbidities, can serve as viable alternatives for other more complex surgical coverage options, as well as can be useful for certain salvage procedures where partial flap or total flap loss is encountered and soft tissue coverage of exposed structures (e.g., nerve, tendon, blood vessels and bone) is required.

\section{Disclaimer}

The opinions expressed in this article are those of the authors and do not necessarily reflect the views of Future Medicine Ltd, the Department of Defense and/or its subsidaries, or the US government.

\section{Financial \& competing interests disclosure}

The authors have no relevant affiliations or financial involvement with any organization or entity with a financial interest in or financial conflict with the subject matter or materials discussed in the manuscript at the time of manuscript submission. This includes employment, consultancies, honoraria, stock ownership or options, expert testimony, grants or patents received or pending, or royalties.

No writing assistance was utilized in the production of this manuscript. 